\title{
Optimización de los niveles de inventario para mejorar los procesos productivos de una organización.
}

\section{Optimization of inventory levels to improve the production processes of an organization.}

\author{
Cont. Rubén Alberto Mackay Véliz ${ }^{1 *}$, Lcd. Teodoro Antonio Escalante \\ Ramírez ${ }^{2}$ \\ 1.* Magister en Administración, Instituto Técnico Bolivariano, Guayaquil, Ecuador. Email: rmackay@itb.edu.ec \\ ORCID: https://orcid.org/0000-0001-9272-3991 \\ 2. Magister en Administración de Empresas Mención en Sostenibilidad en Sectores, Universidad de Guayaquil, Guayaquil, \\ Ecuador. Email: teodoro.escalanter@ug.edu.ec ORCID: https://orcid.org/0000-0002-3682-3098
}

Destinatario: rmackay@itb.edu.ec

Recibido: 21/ enero/2021 Aceptado: 22/febrero/2021 Publicado: 31/marzo/2021

Como citar: Mackay Véliz, R. A., \& Escalante Ramírez, T. A. (2021). Optimización de los niveles de inventario para mejorar los procesos productivos de una organización. Revista E-IDEA Journal of Business Sciences, 3(9), 36-47. https://doi.org/10.53734/eidea.vol3.id86

Resumen: Actualmente las empresas tienen que estar al tanto de los cambios constantes en el mercado global debido a los avances tecnológicos, pero también producto de las exigencias de los consumidores como los son la calidad, precios razonables, mejores servicios y atención personalizada. Es por esto, que constantemente éstas están buscando estrategias y métodos más efectivos para producir los mejores productos o para ofrecer los mejores servicios, para lo cual es indispensable tener un óptimo manejo de sus inventarios. El objetivo general de esta investigación estudiar la importancia que tiene la optimización de los niveles de inventario para mejorar los procesos productivos de las organizaciones. Como conclusión se puede mencionar que Los inventarios son útiles ya que prevén la escasez. Siempre será mejor ahorrar productos que dinero en efectivo por la rentabilidad que genera, permite obtener ganancias adicionales cuando hay alzas de precios, entre otros. A pesar de esto, trae como consecuencia una inmovilización de recursos financieros que podrían usarse mejor en otras actividades con mayor rentabilidad, es decir, podría optarse por mejor uso de los recursos financieros y optimizar así las utilidades.

Palabras Clave: Optimización, gestión de inventario, niveles de inventario.

Abstract: Currently companies have to be aware of the constant changes in the global market due to technological advances, but also a product of consumer demands such as quality, reasonable prices, better services and personalized attention. This is why they are constantly looking for more effective strategies and methods to produce the best products or to offer the best services, for which it is essential to have an optimal management of their inventories. The general objective of this research is to study the importance of optimizing inventory levels to improve the productive processes of organizations. As a conclusion it can be mentioned that Inventories are useful as they anticipate shortages. It will always be better to save products than cash for the profitability it generates, it allows you to obtain additional profits when there are price increases, among others. Despite this, it results in an immobilization of financial resources that could be better used in other activities with greater profitability, that is, it could be opted for a better use of financial resources and thus optimize profits.

Keywords: Optimization, inventory management, inventory levels.

Mackay Véliz, R. A., \& Escalante Ramírez, T. A. (2021). Optimización de los niveles de inventario para 


\section{INTRODUCCIÓN}

$\mathrm{L}$ os grandes cambios tecnológicos en los procesos productivos, así como las mejoras en la productividad y la gestión y control de las actividades económicas, producto de la globalización, han impulsado para que existan actualizaciones en los modelos de gestión.

Es así como, las organizaciones desde el punto de vista de la administración buscan estrategias para maximizar sus beneficios económicos, resaltar las ventajas competitivas, creando valor y fortaleciendo sus finanzas. Actualmente, se han producido grandes cambios en las tecnologías de la información y la comunicación que obviamente han generado cambios en lo administrativo y en lo económico.

Por tanto, la función de la gerencia es vital para el desarrollo de las ventajas competitivas, así como la incorporación de estrategias que faciliten el desarrollo de las capacidades y los recursos y además en el cómo promover procesos que conduzcan al establecimiento de estas ventajas competitivas sostenibles (Garrido y Cejas, M, 2017)

En este orden de ideas, las gestiones de inventario constituyen para las organizaciones una parte sustancial de sus activos, ya que el inventario está vinculado al aumento de la rentabilidad, prediciendo con ello el impacto de las políticas internas de la empresa de acuerdo a los niveles de stock. En este sentido se busca minimizar el costo total de las actividades logísticas que aseguran el servicio que demanda un cliente.

Sobre este contexto, una administración eficaz de inventario puede determinar las ganancias de las organizaciones, al aumentar los ingresos y el total de activos. Ya sean inventarios de materias primas, de producción en proceso o de productos terminados, la gestión de inventario siempre busca reducir al mínimo posible los niveles de existencias y asegurar el suministro del producto en el momento adecuado al área deproducción o al cliente. (Suárez, 2012)

Ahora bien, el presente artículo tiene como objetivo estudiar la importancia que tiene la optimización de los niveles de inventario para mejorar los procesos productivos de las organizaciones y para ello se destacara la gestión de inventario en la empresa, se describirán los modelos de gestión de inventarios que sustentan los procesos administrativos y finalmente se analizara la optimización de los niveles de inventarios en las organizaciones.

Para llevar a cabo lo anteriormente expuesto, se hizo el análisis documental recurriendo a la revisión bibliográfica, y tomando aportes de diferentes investigadores.

El análisis de la importancia de la optimización de los niveles de inventario como oportunidad de mejora en las organizaciones se justifica debido a que gran parte de las empresas carecen de una correcta administración de sus inventarios porque creen que es un gasto y desconocen que esta actividad es efectiva. Si se cuenta con un buen sistema de 
manejo de inventario, se conocen los costos de producción, se evitan desperdicios y se refleja en ganancias para la empresa.

\section{METODOS}

La metodología empleada en esta investigación se basa en un diseño bibliográfico de tipo documental a través de una revisión sistemática de artículos científicos indexados en bases de datos científicas con verificable rigor académico basados en temas de Optimización de niveles de inventario y procesos productivos, se concreta exclusivamente en la recopilación de información de diversas fuentes, con el objeto de organizarla describirla e interpretarla de acuerdo con ciertos procedimientos que garanticen confiabilidad y objetividad en la presentación de los resultados (Palella Stracuzzi y Martins Pestana, 2010).

\section{DESARROLLO}

\section{La Gestión de Inventario en las Empresas}

El inventario según Westreicher G (2020) es el registro de los bienes que pertenecen a una persona natural o jurídica. En otras palabras, el inventario, es un documento donde se anotan todas las pertenencias del individuo o empresa. Esto, da constancia de los activos con los que cuenta la empresa, con fines contables o de otra naturaleza.

Generalmente se refiere el inventario a las existencias de una empresa, allí se registran las materias primas, los bienes o productos intermedios y los productos finales que ofrece la empresa a sus clientes.

En este sentido, el inventario en una empresa manufacturera está conformado por la materia prima, insumos o productos en proceso y posterior comercialización en productos terminados. En las empresas comerciales está compuesto por el conjunto de mercancía y artículos terminados destinados para la venta. En lo que respecta a las empresas de servicio, el inventario está integrado por todo el suministro necesario para su funcionamiento y prestación de servicio (Durán, 2012)

La gestión de inventario según Westreicher G (2019) es el indicador respecto al ingreso y salida de insumos, productos terminados, bienes auxiliares y herramientas que posee una empresa, siendo fundamental para optimizar las operaciones de la compañía.

De igual manera, la cadena de suministro Roldán (2017) es el conjunto de actividades, servicios y medios de distribución necesarios para llevar a cabo el proceso de venta de un producto en su totalidad, abarcando desde la gestión de materia prima, su transformación, su fabricación, transporte y entrega a los clientes.Entonces, la cadena de suministro es una función logística y estratégica que abarca todas las operaciones que se llevan a cabo para que una mercancía logre llegar al cliente en buenas condiciones.

Mackay Véliz, R. A., \& Escalante Ramírez, T. A. (2021). Optimización de los niveles de inventario para mejorar los procesos productivos de una organización. E-IDEA Journal of Business Sciences, 3(9), 36-47. 
Ahora bien, la cadena de suministro tiene como objetivo principal citando a Roldán (2017) satisfacer las necesidades del cliente final de la mejor manera posible. Para ello las empresas deben entregar los productos y servicios a tiempo, evitar las pérdidas innecesarias, establecer canales de comunicación y coordinación efectivos, manejar de manera correcta los inventarios y almacenes y estar atentas a los cambios en las ofertas y demandas y del entorno en general.

\section{Tipos de Inventarios}

Los inventarios tienen como función flexibilizar las operaciones administrativas de esta manera los inventarios de productos se convierten en una necesidad ya que permite oportunidades de desarrollo y expansión en los procesos. De igual manera, otra de las funciones en la gestión de inventario está vinculada a la eliminación de irregularidades en la compra o producción en lotes en exceso. Con esto se lograría la administración eficiente e integral de la empresa.

En la actualidad muchos especialistas-(Mora y Martiliano 2014; Suárez 2012; Heizer y Barry, 2012) coinciden en la tipología de diferentes inventarios, en la Figura 1 se resumen e identifican algunos de estos:

\section{Figura 1}

\section{Tipos de Inventarios.:}

\begin{tabular}{|c|l|}
\hline Inventario Perpetuo & Es aquel que se lleva en continuo acuerdo con las exigencias en el almacén. \\
\hline Intermitentes & Este inventario se puede efectuar varias veces al ano \\
\hline Final & Este inventario se realiza al término del ejercicio económico, \\
\hline Inicial & Se realiza al dar comienzo de las operaciones. \\
\hline Fisico & Es el inventario Real \\
\hline Mixto & Pertenece a una clase determinada de mercancia \\
\hline Productos Terminados & Son para todas las mercancias. \\
\hline Tránsito & $\begin{array}{l}\text { Son utilizados para sostener las operaciones y abastecer los conductos de las } \\
\text { empresas con sus proveedores y clientes }\end{array}$ \\
\hline Materia Prima & Representan existencias de los insumos básicos \\
\hline En Procesos & $\begin{array}{l}\text { Son los que se tienen a medida que se anade mano de obra, otras materias y de más } \\
\text { costos indirectos }\end{array}$ \\
\hline Consignación & $\begin{array}{l}\text { Refiere la mercaderia que se entrega para ser vendida pero el titulo de propiedad lo } \\
\text { conserva el vendedor }\end{array}$ \\
\hline Máximo & Es aquel que se mide en meses de demanda pronosticada. \\
\hline Inventario Minimo & Es la cantidad minima del inventario a ser mantenida en el almacén. \\
\hline Disponible & Aquel que está a disposición para la venta con productos terminados. \\
\hline Inventario en Línea & Es aquel que aguarda a ser procesado en la linea de producción. \\
\hline Agregado & $\begin{array}{l}\text { Se aplica para administrar la exigencia del único articulo que representa un alto costo, } \\
\text { para minimizar el impacto de este costo. }\end{array}$ \\
\hline Cuarentena & Debe cumplir con un periodo de almacenamiento \\
\hline Previsión & Se tienen para cumplir con una necesidad futura y definida. \\
\hline
\end{tabular}

Fuente: (Garrido y Cejas, M, 2017) a partir de (Suárez, 2012)

De manera similar, en opinión de Noori y Radford (1997) los inventarios se pueden clasificar por su forma y función.

Según su forma los inventarios se clasifican en: 


\section{E-IDEA}

JOURNAL OF BUSINESS SCIENCES

- Inventario de materia prima (MP): son materiales básicos e insumos que ingresan al proceso.

- Inventario de producto en proceso (PP): son materiales en proceso de producción.

- Inventario de producto terminado (PT): son los materiales que han pasado por los procesos productivos y que serán destinados a su comercialización o entrega a los clientes

Mientras que según su función se clasifican en:

- $\quad$ Inventario de Seguridad o de Reserva: es el que se mantiene para suplir los riesgos de paradas no planeados de la producción o inesperados aumentos en la demanda de los clientes.

- Inventario de desacoplamiento: es el que se requiere entre dos operaciones inmediatas cuyas tasas de producción no pueden acoplarse logrando que el proceso funcione.

- Inventario en tránsito: es el movimiento de los materiales que avanzan en la cadena de valor. Estos materiales son artículos que se han pedido, pero no se han recibido todavía y se trasladan de los proveedores a las empresas.

- Inventario de ciclo, resulta cuando la cantidad de unidades compradas (o producidas) con el fin de reducir los costos por unidad de compra (o incrementar la eficiencia de la producción) es mayor que las necesidades inmediatas de la empresa.

- Inventario de Previsión o Estacional, se acumula cuando una empresa elabora más de lo necesario durante los periodos de demanda baja para satisfacer las de demanda alta.

\section{Tipos de Gestión de Inventarios para su valoración}

Según Westreicher G (2019) las formas de calcular el costo de una mercancía son:

- $\quad$ Método de Valoración FIFO:este método estima que todo lo que primero ha entrado en el almacén debe ser lo que primero salga o se le dé salida. Es decir, se toma en cuenta que los insumos que van siendo utilizados primero son los que tienen mayor tiempo en el almacén.

- $\quad$ Método de valoración LIFO:Y es menos usado que el FIFO y es ideal para cuando se almacena productos no perecederos. En este caso la materia prima comprada recientemente es la que se usa de una vez para elaborar el producto.

- $\quad$ Precio medio ponderado (PMP): consiste en calcular un promedio entre el valor de los insumos o materia prima que se usan al inicio del proceso y el de aquellos que fueron ingresando mientras se daba la elaboración el producto final.

Mackay Véliz, R. A., \& Escalante Ramírez, T. A. (2021). Optimización de los niveles de inventario para mejorar los procesos productivos de una organización. E-IDEA Journal of Business Sciences, 3(9), 36-47. 


\section{La optimización de los inventarios en la mejora de la productividad. Importancia de la gestión de inventarios}

En el caso del inventario Castillo (2018) afirma que hay que estar atentos a que el nivel de este no sea tan elevado que se traduzca en un costo por tener paralizado un capital que podría emplearse en otra actividad, de igual forma, muy poco generaría que la empresa produzca sobre pedido, situación igualmente desfavorable porque debe satisfacer de inmediato las demandas de los clientes. Por eso se hace necesario que la empresa tenga determinado el nivel apropiado de inventarios de manera que haya equilibrio entre estos dos extremos.

Según Westreicher G (2019) la importancia de la gestión de inventarios radica en que permite calcular con precisión el costo de producir los bienes, permite también calcular la producción necesaria y así abastecer la demanda esperada por los clientes, se reduce el costo de mantenimiento de inventario y hasta se puede identificar y detectar si en alguna parte de la cadena o proceso

\section{Niveles de Inventarios}

El nivel de inventario según Bind ERP (2020) es el control de la cantidad óptima de mercancía para evitar la venta perdida o costo de oportunidad, así como el exceso de productos. Para lograr un nivel de inventario óptimo, es necesario desarrollar un alto nivel de confiabilidad y sincronía entre las distintas áreas de la empresa; definir la forma en que se repondrán los productos y mantener la información en tiempo real.

En atención a lo anterior, Handerson (2020) define cada nivel de inventario como:

- $\quad$ Nivel Mínimo: Se deben mantener los niveles mínimos de existencias para garantizar los materiales y dar continuidad a la producción. Si no se alcanza el nivel mínimo, se suspenden las operaciones. Para asegurar el nivel mínimo deben tener en cuenta los siguientes elementos de la cadena de suministro: tiempo de entrega, tasa de consumo, naturaleza del material, nivel de reordenamiento.

- $\quad$ Nivel Máximo: una empresa no debe sobrepasar el nivel máximo para evitar el exceso de inventario. El exceso de inventario puede provocar además de desorganización, altos costos de mantenimiento y mayor probabilidad de disconformidades en las existencias.

La determinación de un nivel máximo de existencias depende de: Capital disponible, espacio en almacén, tasa de consumo, costo de mantenimiento, disponibilidad de materiales y cambios de demandas.

- $\quad$ Nivel de peligro: de llegar a este nivel, indica que el suministro de inventario está cerca de agotarse y las empresas deben evitar llegar a este nivel. Si se alcanza el nivel de peligro, la empresa debería considerar la posibilidad de acelerar más materiales. 


\section{E-IDEA}

J OURNAL OF BUSINESS SCIENCES

- $\quad$ Nivel medio: el nivel medio de stock es la cantidad promedio de stock que existe en el almacén en un momento dado.

\section{La optimización de los inventarios}

Es vital que las empresas optimicen sus inventarios ya que de existir fallas en la logística puede fracasar y cerrar. Por eso cuando los procesos productivos no están bien gestionado, se genera un alto nivel de desperdicio, altos costos por trabajos, errores en el control del inventario, entre otros inconvenientes, trayendo como consecuencia poca o nula rentabilidad (Conexión Esan, 2017).

Específicamente Conexión Esan (2017) menciona que las claves para manejar la optimización de inventarios son

- Clasificar las mercancías en tres categorías como mínimo, para tener una mejor visión y administración del inventario.

- Crear una política de almacén. El objetivo es tener en claro detalles como el número de productos que debe haber, las fechas de auditorías, los tiempos para realizar entregas.

- Los procesos deben ser automatizados. Esto es necesario cuando la empresa ya está en constante crecimiento y se hace más difícil gestionar un inventario de gran tamaño y movimiento.

De igual manera Rizo (2019) comenta que para optimizar el manejo de inventarios en las empresas se debe tomar en cuenta lo siguiente:

a) Hacer un pronóstico de la demanda: se debe evitar tener exceso de mercancía o desabastecimiento y para eso hay que hacer pronósticos de la demanda y llevar un buen registro de la rotación de los productos que vende.

b) Diseñar una política de inventarios: ya que en ella se puede definir el nivel de producto terminado que debe tener siempre en almacén, la clasificación de cada tipo de mercancía y su rotación, los tiempos para realizar pedidos, fechas en las cuáles se deben realizar auditorías y revisiones periódicas, etc. El tener una política de inventario dará directrices a la empresa y así generar una mejora continua en el manejo del inventario.

c) Automatizar los procesos: haciendo uso de la tecnología, aprovechar los sistemas administrativos que están diseñados para el control de los inventarios. Estos sistemas permiten llevar información de las entradas y salidas de mercancía.

d) Hacer equipo con los proveedores: tener precisión de cuáles son los proveedores que más impactan en tu inventario y en las operaciones de la empresa, para planificar con ellos estrategias en tiempos normales $y$ en situaciones extraordinarias.

e) Maximizar los márgenes: gestionar el control de inventario es fundamental para alcanzar los objetivos de los ingresos obtenidos por ventas y así invertir en el crecimiento del negocio. 
f) Monitorear: se debe disponer de buenos controles y así monitorear de forma permanente los niveles de inventario para que en caso de que ocurra una falla se detecte y se dé solución oportunamente (Rizo, 2019)

\section{Sistema de Clasificación $A B C$ de los inventarios}

El análisis $\mathrm{ABC}$ diseñado por General Electric durante los años 50 del siglo pasado, permite a las organizaciones separar sus artículos de inventario en tres clases: A, B, C y es útil cuando las empresas venden cientos de artículos. La clasificación $\mathrm{ABC}$ en opinión de (Wiston, 2005) explica que un pequeño porcentaje del total de productos representan un gran porcentaje del valor en dólares de la inversión. Los productos "A", muy importantes, concentran la máxima inversión. El grupo "B", moderadamente importantes, está formado por los artículos que siguen a los "A" en cuanto a la magnitud de la inversión. Al grupo "C", menos importantes, lo componen una gran cantidad de productos que solo requieren de una pequeña inversión. Esta clasificación se puede apreciar en la figura 2.

\section{Figura 2}

Clasificación ABC de los inventarios:

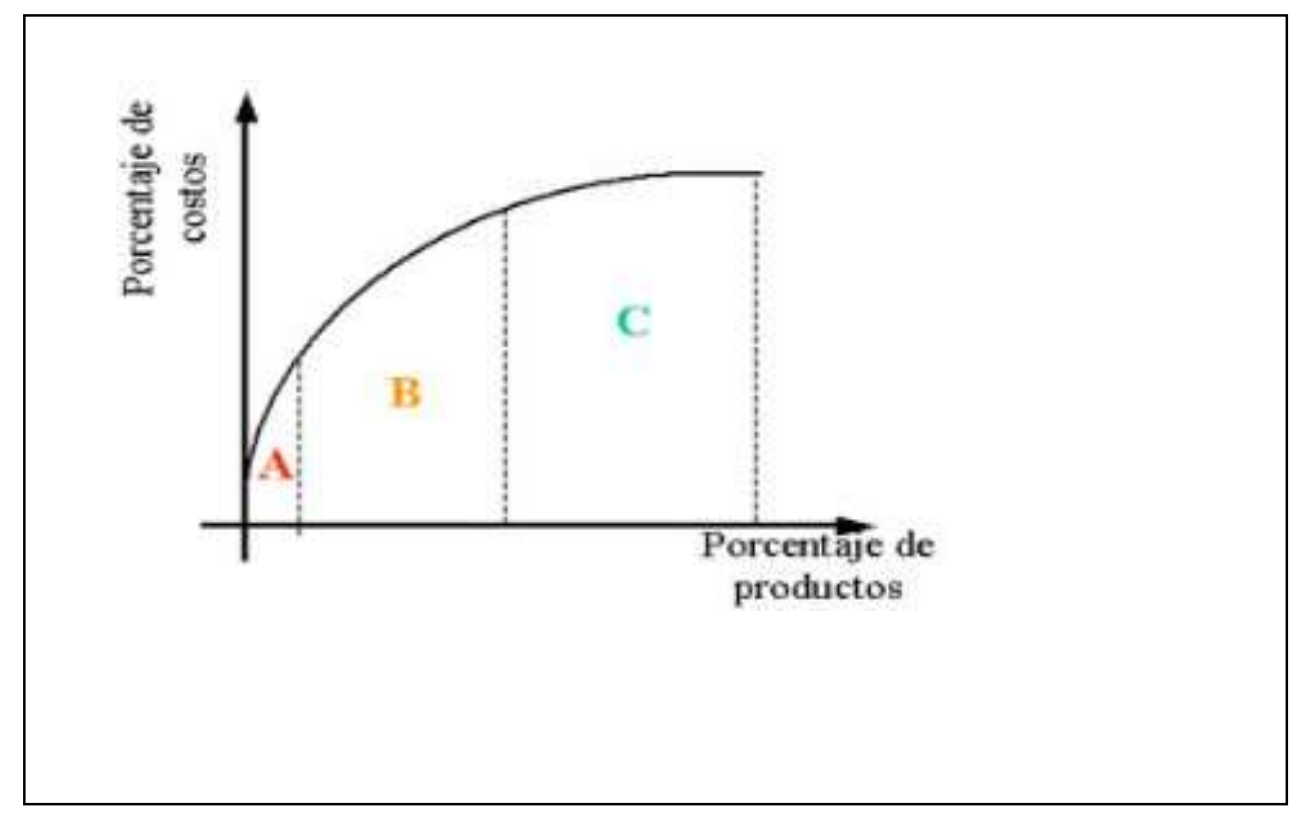

Fuente: (Castillo, 2018)

El control de los productos "A" merece la mayor atención y cuidado en su administración dada la magnitud de la inversión, en tanto los productos "B" y "C" estarán sujetos a procedimientos de control menos estrictos.

Just In Time (IT) 
El justo a tiempo o Just in Time lo define (Hernández, 2002) como una técnica japonesa para rediseñar organizaciones, cuyo objetivo es disminuir la inversión en almacenes y en proceso productivo. El ideal del JIT es cero inventarios. Implica una coordinación muy grande con proveedores, en ocasiones obligándolos a que pongan sus fábricas pegadas a la empresa. Tiene por objeto evitar pérdidas por sobreinversión en inventarios y por obsolescencia de materias primas o refacciones y desperdicios de materia prima.

Con esta técnica se busca optimizar los niveles de inventarios ya que fomenta el mejoramiento continuo para así obtener la máxima eficiencia y eliminar el gasto excesivo de cualquier forma en todas las áreas de la organización, sus proveedores y clientes (Castillo, 2018)

Los procesos de producción en un sistema de JIT buscan elevar la productividad mediante la eliminación del desperdicio. La eliminación de actividades que no agregan valor es elemento principal del sistema. De acuerdo a la filosofía del JIT los inventarios son un desperdicio ya que requieren capital, almacenamiento y mano de obra.

\section{CONLUSIONES}

Los inventarios son útiles ya que prevén la escasez. Siempre será mejor ahorrar productos que dinero en efectivo por la rentabilidad que genera, permite obtener ganancias adicionales cuando hay alzas de precios, entre otros. A pesar de esto, trae como consecuencia una inmovilización de recursos financieros que podrían usarse mejor en otras actividades con mayor rentabilidad, es decir, podría optarse por mejor uso de los recursos financieros y optimizar así las utilidades.

El inventario es por lo general, el mayor activo en el balance de una empresa Garrido y Cejas (2017) y como consecuencia, los costos generados por inventarios representan uno de los mayoresrubros que se reflejan en el estado de resultados, en este sentido cuando se evalúan lascuentas relacionadas con los inventarios- como forma básica- están presentes lossiguientes componentes: Inventarios (inicial), Compras, Devoluciones en Compras,Gastos de Compras ,Ventas, Devoluciones enVentas, Mercancías en Tránsito e inventario final.

El objetivo general de la gestión de inventarios es garantizar la disponibilidad oportuna de los elementos que se necesitan (materia prima, materiales en proceso, productos terminados, insumos, repuestos, etc.), en las condiciones deseadas y en el lugar correcto para así lograr una ventaja competitiva.

Teniendo en cuenta que la gestión de inventarios es una actividad transversal a la cadena de suministro, deben implementarse estrategias para lograr un manejo efectivo del mismo con el fin evitar consecuencias no deseadas, como el efecto látigo, un bajo nivel de servicio y el incremento de costos de administración de inventarios (Salas, Maiguel, H, y Acevedo, J, 2017)

Mackay Véliz, R. A., \& Escalante Ramírez, T. A. (2021). Optimización de los niveles de inventario para mejorar los procesos productivos de una organización. E-IDEA Journal of Business Sciences, 3(9), 36-47. 
La gestión se encarga de mantener la productividad en las operaciones relacionadas con la administración del inventario, mientras que la optimización se ocupa de incrementar las ganancias de la empresa provenientes del uso y manejo de este.El inventario es parte medular de las operaciones de las empresas. Si no se gestiona de forma adecuada, puede traer consecuencias negativas como la pérdida de ventas, estancamiento en la producción, retrasos y pérdidas financieras. (Rizo, 2019). 


\section{E-IDEA}

J OURNAL OF BUSINESS SCIENCES

\section{REFERENCIAS BIBLIOGRÁFICAS}

Bind ERP. (2020). Nivel de Inventario o Stock. Recuperado el 20 de Junio de 2021, de https://www.bind.com.mx/Glosario/Definicion/37-nivel-de-inventario-de-stock

Castillo, G. (2018). Niveles de Inventarios. Universidad de la Americas Puebla. Recuperado el 19 de Junio de 2021, de http://catarina.udlap.mx/u_dl_a/tales/documentos/lmnf/castillo_g_ka/capitulo1.p df

Conexión Esan. (2017). ¿Como mejorar la gestión de tus inventarios? Recuperado el 20 de Junio de 2021, de https://www.esan.edu.pe/apuntesempresariales/2017/12/como- mejorar-la-gestion-de-tus-inventarios/

Durán, Y. (2012). Administración del inventario: elemento clave para la optimización de las utilidades en lasempresas. Visión Gerencial , 55-78.

Garrido, I., \& Cejas, M. (2017). La Gestión de Inventario como Factor Estratégico en la Administración de Empresas. Revista Negotium , 109-129.

Handerson, C. (2020). Como calcular los niveles de inventario y mantenerlos bajos. Zip ordering. Recuperado el 20 de Junio de 2021, de https://zipordering.com/es/economic- order-quantity/inventory-level.html

Heizer, \& Barry. (2012). Direccion de la Produccion . México: Ediciones Prentice Hall.

Hernández, S. (2002). Adminsitracion: Pensamiento, proceso, estrategia y vanguardia. México: Mc Graw Hill. 
Mora, ., \& Martiliano,. (2014). Modelos de la Optimizacion de la Gestion Logistica. Bogota: Ediciones ECOE.

Noori, H., \& Radford, R. (1997). Administración de Operaciones y Producción. Colombia: Mc Graw Hill.

Rizo, J. (2019). Consejos para la optimizar el manejo de inventarios en una PYME. ContadorMX. Recuperado el 20 de Junio de 2021, de https://contadormx.com/2019/11/21/6-consejos-para-la-optimizacion-en-elmanejo-de- inventarios-en-una-pyme/

Roldán, P. (25 de abril de 2017). Cadena de Suministro. Economipedia.com. Recuperado el 19 de Junio de 2021, de https://economipedia.com/definiciones/cadena-desuministro.html\#referencia

Salas, K., Maiguel, H, \& Acevedo, J. (2017). Metodología de Gestión de Inventarios para determinar los nivelesde integración y colaboración en una cadena de suministro. Ingeniare. Revista Chilena de Ingenieria , 326 - 337.

Suárez, C. (2012). Gestion de Inventarios. Colombia: Ediciones de la U.

Westreicher, G. (2019). Gestión de Inventarios. Obtenido de https://economipedia.com/definiciones/gestion-de-inventarios.html\#referencia

Westreicher, G. (2020). Inventario. Economipedia. Recuperado el 20 de Junio de 2021, de https://economipedia.com/definiciones/inventario.html\#referencia

Wiston, W. (2005). Investigación de Operaciones- Aplicaciones y Algoritmos. México: Thomson. 